\title{
The potential use of microcalorimetry in rapid differentiation between septic arthritis and other causes of arthritis
}

\author{
E. Yusuf • T. Hügle • T. Daikeler • C. Voide • O. Borens • \\ A. Trampuz
}

Received: 22 July 2014 / Accepted: 10 September 2014

(C) Springer-Verlag Berlin Heidelberg 2014

\begin{abstract}
Current diagnostic methods in differentiating septic from non-septic arthritis are time-consuming (culture) or have limited sensitivity (Gram stain). Microcalorimetry is a novel method that can rapidly detect microorganisms by their heat production. We investigated the accuracy and time to detection of septic arthritis by using microcalorimetry. Patients older than 18 years of age with acute arthritis of native joints were prospectively included. Synovial fluid was aspirated and investigated by Gram stain, culture and microcalorimetry. The diagnosis of septic arthritis and non-septic arthritis were made by experienced rheumatologists or orthopaedic surgeons. Septic arthritis was diagnosed by considering the finding of acute arthritis together with findings such as positive Gram stain or positive culture of synovial fluid or positive blood culture. The sensitivity and specificity for diagnosing septic arthritis and the time to positivity of microcalorimetry were determined. Of 90 patients (mean age 64 years), nine had septic arthritis, of
\end{abstract}

E. Yusuf $\cdot$ C. Voide $\cdot$ A. Trampuz

Infectious Diseases Service, Department of Medicine, University

Hospital, Lausanne, Switzerland

T. Hügle $\cdot$ T. Daikeler

Division of Rheumatology, Department of Internal Medicine,

University Hospital, Basel, Switzerland

O. Borens

Orthopaedic Septic Surgical Unit, Department of Surgery and

Anaesthesiology, University Hospital, Lausanne, Switzerland

Present Address:

E. Yusuf $(\bowtie)$

Department of Medical Microbiology and Infection Control, UZ

Brussel, Laarbeeklaan 101, 1210 Brussels, Belgium

e-mail: angga.yusuf@gmail.com

Present Address:

A. Trampuz

Center for Musculoskeletal Surgery, Charité, University Medicine,

Free and Humboldt-University of Berlin, Berlin, Germany whom four (44\%) had positive Gram stain, six (67\%) positive synovial fluid culture and four (44\%) had positive blood culture. The sensitivity of microcalorimetry was $89 \%$, the specificity was $99 \%$ and the mean detection time was $5.0 \mathrm{~h}$ (range, 2.2-8.0 h). Microcalorimetry is an accurate and rapid method for the diagnosis of septic arthritis. It has potential to be used in clinical practice in diagnosing septic arthritis.

\section{Introduction}

Septic arthritis of native joints is a medical emergency and is associated with substantial morbidity and mortality [1, 2]. Immediate intervention (i.e. arthroscopic lavage) and antimicrobial treatment are required in order to prevent irreversible damage of the joint. Therefore, septic arthritis needs to be rapidly and accurately differentiated from non-septic causes of acute arthritis, such as crystal-induced or rheumatic disorders, where invasive intervention is not needed or can even worsen the condition $[2,3]$.

Current diagnostic methods of septic arthritis have insufficient accuracy or are time-consuming. Gram stain of synovial fluid has limited sensitivity (about $50 \%$ ) and culture of synovial fluid requires at least $24 \mathrm{~h}$ of incubation $[4,5]$. Other diagnostic methods, such as white blood cells and serum Creactive protein [6], cannot differentiate between septic and non-septic causes of arthritis. Synovial fluid leucocyte count can differentiate septic from non-septic arthritis only when a very significant amount of leucocytes is present $[7,8]$.

Microcalorimetry is a novel technique that measures heat production produced by all living cells due to their metabolisms [9]. However, in contrast to leucocytes and other host cells, only microorganisms cause an exponential increase in heat production due to cell replication. Therefore, this technique can be used to differentiate septic from non-septic arthritis. Its use have been investigated in other fields of 
medicine, where the rapid detection of microorganisms is crucial, for example in detecting the presence of microorganisms in blood transfusions product [10]. Microcalorimetry has also been evaluated for the rapid determination of antimicrobial susceptibility of bacteria and fungi [11-13]. An extensive review on microcalorimetry in microbiology was recently published [9].

The aim of this study was to assess the accuracy and speed of microcalorimetry in diagnosing septic arthritis. An accurate and rapid method for microbial detection can initiate early treatment and can prevent unnecessary invasive interventions in septic arthritis and can also prevent the unnecessary use of antibiotics in patients with non-septic arthritis.

\section{Patients and methods}

\section{Study population}

The study was performed in two university hospitals in Switzerland (Lausanne and Basel) between January 2006 and May 2010. Patients older than 18 years of age who presented in the emergency department with clinical suspicion of joint sepsis, defined as the occurrence of acute signs of inflammation of the native joint (redness, warmth, effusion or pain), were prospectively included. Demographic, clinical, laboratory and microbiology data were collected using a standardised case report form. The study was approved by the institutional review boards of the participating study centres. Written informed consent was obtained from all the participants.

\section{Definition}

The diagnoses of septic arthritis and non-septic arthritis were made by experienced rheumatologists or orthopaedic surgeons. Septic arthritis was diagnosed by considering the finding of acute arthritis together with findings such as positive Gram stain, positive culture of synovial fluid and positive blood culture [5]. Non-septic arthritis was diagnosed by using serological tests, polarisation microscopy, histopathology and imaging according to standard rheumatologic and orthopaedic practice.

\section{Specimen collection}

Synovial fluid was aspirated by the rheumatologist or orthopaedic surgeon using a standardised aseptic technique. One millilitre of the fluid was immediately transferred into a 4-ml microcalorimetry vial pre-filled with $2 \mathrm{ml}$ of tryptic soy broth. The remaining fluid was transferred into a native vial for Gram stain and culture, and, whenever possible, into an EDTA (ethylenediaminetetraacetic acid) tube for the determination of leucocyte counts. In addition, two to three pairs of blood cultures were collected in all patients with temperature $>38^{\circ} \mathrm{C}$ before any antibiotic treatment was started.

Standard microbiology methods

A quantity of $0.1 \mathrm{ml}$ of synovial fluid was inoculated on agar plates and incubated aerobically for 5 days with $5 \% \mathrm{CO}_{2}$ and anaerobically for 7 days at $37{ }^{\circ} \mathrm{C}$. In addition, $0.5 \mathrm{ml}$ of synovial fluid was inoculated in thioglycolate broth and the residual volume was inoculated into a BacT/ALERT anaerobic blood culture bottle (bioMérieux, Marcy L'Etoile, France) and incubated for 7 days. Blood culture bottle pairs were placed into the BacT/Alert 3D blood culture instrument and incubated for 5 days or until they signalled positive for growth. Signal-positive bottles were handled according to standard laboratory protocols for identification. Identification was performed using standard biochemical microbiological techniques or Microflex LT MALDI-TOF (Bruker Daltonics, Bremen, Germany).

\section{Microcalorimetry}

Ampoules inoculated with $1 \mathrm{ml}$ of synovial fluid were inserted into an isothermal microcalorimeter equipped with 48 measuring channels (thermal activity monitor, model 3102 TAM III; TA Instruments, New Castle, DE, USA), first in the equilibration position for $15 \mathrm{~min}$ to reach $37^{\circ} \mathrm{C}$ and then for another $30 \mathrm{~min}$ to obtain an accurate measurement of heat flow. Heat production was then measured continuously and expressed as heat flow over time in microwatts $(\mu \mathrm{W})$. The sensitivity of the microcalorimeter provided by the manufacturer was $0.225 \mu \mathrm{W}$ t.

The detection time was defined as the time from the insertion of an ampoule into the microcalorimeter until exponential growth produced a rising heat flow rate signal and a heat flow $10 \mu \mathrm{W}$ (i.e. 40 times the effective sensitivity of the calorimeters) above the lowest point of the power-time curve was observed [10].

The readers of the microcalorimetry results were blinded to the clinical and other laboratory results of the patients. No repeated reading to assess the reproducibility of microcalorimetry was performed.

Statistical analysis

The mean along with the standard deviation (SD) for variables were calculated. A $2 \times 2$ contingency table was used to calculate the sensitivity and specificity of microcalorimetry compared to the diagnosis of septic arthritis. Fisher's exact test was used to compare categorical variables of synovial fluid and blood (i.e. positivity of Gram stain, microcalorimetry and blood culture) of septic and non-septic arthritis patients. Continuous synovial fluid variables (i.e. leucocyte count and 
percentage of neutrophils) of septic arthritis were compared with those of non-septic arthritis using the Mann-Whitney $U$ test, since Shapiro-Wilk tests did not show normal distributions of these variables. Differences were considered to be statistically significant when the $p$-value was $<0.05$. All analyses were performed using SPSS Statistics version 20.0 (IBM Corp., Armonk, NY, USA).

\section{Results}

Characteristics of septic and non-septic arthritis

Ninety patients with acute arthritis were included, with a mean age of $64 \pm 18$ years and $50(56 \%)$ were males (Table 1$)$. The most frequently affected joint was the knee (73\%). Septic arthritis was diagnosed in nine patients, involving the knee $(n=5)$, hip $(n=2)$ and shoulder $(n=2)$. The synovial fluid culture grew Staphylococcus aureus $(n=3)$, Streptococcus spp. $(n=2)$, Morganella morganii $(n=1)$ and was negative in three patients. The blood culture was positive for Streptococcus spp. in these three septic arthritis patients with negative synovial fluid culture. Only in one out of nine patients with septic arthritis was the blood culture positive with S. aureus. Gram stain was positive in four out of nine patients (44\%) with septic arthritis (Table 2). All cultures of synovial fluid and blood cultures required more than $24 \mathrm{~h}$ to reach positivity.

Among 81 patients with non-septic arthritis, none had positive Gram stain, three had contamination of synovial fluid culture (coagulase-negative staphylococci in two and Corynebacterium diphtheriae in one patient) and one had positive microcalorimetry (Table 2). The synovial fluid leucocyte count and percentage of neutrophils were available in all patients with septic arthritis and in 49 of 81 patients with non-septic arthritis. The leucocyte count and percentage of neutrophils did not differ between patients with septic and non-septic arthritis: $18.6 \times 10^{3} / \mathrm{mm}^{3}$ versus $15.3 \times 10^{3} /$ $\mathrm{mm}^{3}, p=0.38$, and $88.9 \%$ versus $78.5 \%, p=0.45$, respectively.

\section{Microcalorimetry of synovial fluid}

Of nine patients with septic arthritis, eight were positive by microcalorimetry. The heat flow of positive microcalorimetry is shown in Fig. 1. Microcalorimetry had a sensitivity of $89 \%$ and a specificity of $99 \%$ in diagnosing septic arthritis. One patient with false-positive microcalorimetry results was diagnosed with gout. The mean time to positivity by microcalorimetry was $5.0 \mathrm{~h}$ (range, 2.2 to $8.0 \mathrm{~h}$ ). There was no correlation between detection time and synovial fluid leucocyte count $(p=0.2)$.
Table 1 Characteristics of 90 patients with acute arthritis of the native joint

\begin{tabular}{lc}
\hline Characteristic & \multicolumn{2}{l}{ Value } \\
\hline Mean age \pm SD, years & $64 \pm 18$ \\
No. of males (\%) & $50(56 \%)$ \\
Type of affected joint, no. (\%) & $66(73 \%)$ \\
Knee & $8(9 \%)$ \\
Hip & $7(8 \%)$ \\
Shoulder & $7(8 \%)$ \\
Ankle & $2(2 \%)$ \\
Elbow & \\
Cause of acute arthritis, no. (\%) & $9(10 \%)$ \\
Septic arthritis & $81(90 \%)$ \\
Non-septic joint disease & 17 \\
Gout & 13 \\
Calcium pyrophosphate dehydrate disease & 16 \\
Undifferentiated arthritis & 13 \\
Activated osteoarthritis & 11 \\
Reactive arthritis & 11 \\
Other types of arthritis & \\
\hline
\end{tabular}

$S D$ standard deviation

${ }^{\text {a }}$ Rheumatoid arthritis $(n=4)$, psoriasis-associated arthritis $(n=3)$, inflammatory bowel disease $(n=2)$, paraneoplastic manifestation $(n=1)$, Behcet's disease $(n=1)$

\section{Discussion}

We showed that microcalorimetry of synovial fluid has excellent performance and can be used to differentiate septic from non-septic arthritis. Microcalorimetry also provides faster results than conventional cultures of synovial fluid or blood. The results of the present study were obtained within $8 \mathrm{~h}$. When handling and data analysis were included, the results will be obtained in no more than $9 \mathrm{~h}$. This is comparable to a recent study where microcalorimetry can detect $\sim 10^{5}$ colonyforming units $(\mathrm{CFU}) / \mathrm{ml}$ of bacteria in artificial urine within $7 \mathrm{~h}$ (including the preparation and data processing time) [12].

Because it can provide rapid results [9], microcalorimetry will have benefit in conditions where fast differentiation between infectious and non-infectious causes of a disease is important, for example in differentiating septic from nonseptic arthritis. The excellent specificity and high specificity of microcalorimetry in diagnosing septic arthritis as shown in this study can help the clinician in making a decision for the treatment. Its high specificity of $99 \%$ means that, in case of positive results, we can be $99 \%$ sure that there is septic arthritis. Consequently, arthroscopic lavage and antimicrobial treatment can be started. The sensitivity of microcalorimetry of $89 \%$ however, which means that septic arthritis cannot be excluded with negative calorimetry results. Efforts on increasing the sensitivity of microcalorimetry in the clinical setting is 
Table 2 Laboratory and microbiological results in nine patients with septic and 81 patients with non-septic arthritis

\begin{tabular}{|c|c|c|c|}
\hline Results & Septic arthritis $(n=9)$ & Non-septic arthritis $(n=81)$ & $p$-Value \\
\hline \multicolumn{4}{|l|}{ Synovial fluid } \\
\hline Leucocyte count $\left(\times 10^{3} / \mathrm{mm}^{3}\right)$, mean $\pm \mathrm{SD}$ & $18.6 \pm 20.3$ & $15.3 \pm 21.1$ & 0.38 \\
\hline Neutrophil count $(\%)$, mean \pm SD & $88 . \pm 7.1$ & $78.5 \pm 26.4$ & 0.45 \\
\hline Positive Gram stain, no. (\%) & $4(44 \%)$ & 0 & $<0.001$ \\
\hline Positive culture, no. (\%) & $6(67 \%)^{\mathrm{a}}$ & $3(4 \%)^{b}$ & $<0.001$ \\
\hline Positive microcalorimetry, no. (\%) & $8(89 \%)$ & $1(1 \%)$ & $<0.001$ \\
\hline \multicolumn{4}{|l|}{ Blood } \\
\hline Positive culture, no. (\%) & $4(44 \%)^{\mathrm{c}}$ & 0 & $<0.001$ \\
\hline
\end{tabular}

$S D$ standard deviation

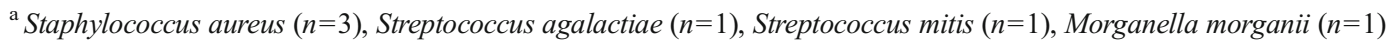

${ }^{\mathrm{b}}$ Coagulase-negative staphylococci $(n=2)$, Corynebacterium spp. $(n=1)$

${ }^{\mathrm{c}}$ Staphylococcus aureus $(n=1)$, Streptococcus agalactiae $(n=1)$, Streptococcus gallolyticus $(n=1)$, Streptococcus mitis $(n=1)$

ongoing [9]. In the setting of septic arthritis, the sensitivity may be increased by using a larger volume of synovial fluid and supplemented growth media. We found one false-positive microcalorimetry result in the present study. It was perhaps caused by contamination, as the culture of medium grew a typical skin bacterium (Corynebacterium spp.). One falsenegative microcalorimetry result was also not diagnosed by synovial fluid culture and by Gram stain, but with blood culture only (Streptococcus mitis). The patient with this false-negative microcalorimetry received antibiotics before joint aspiration.

Our study is the first on the use of microcalorimetry in the diagnosis of septic arthritis; therefore, no comparison with other studies is possible. We recently showed that microcalorimetry of sonication fluid increases the accuracy and, most importantly, led to faster diagnosis of prosthetic joint infection [14]. At present, the obstacles for widespread clinical use of microcalorimetry are the technical aspects (e.g. size of the

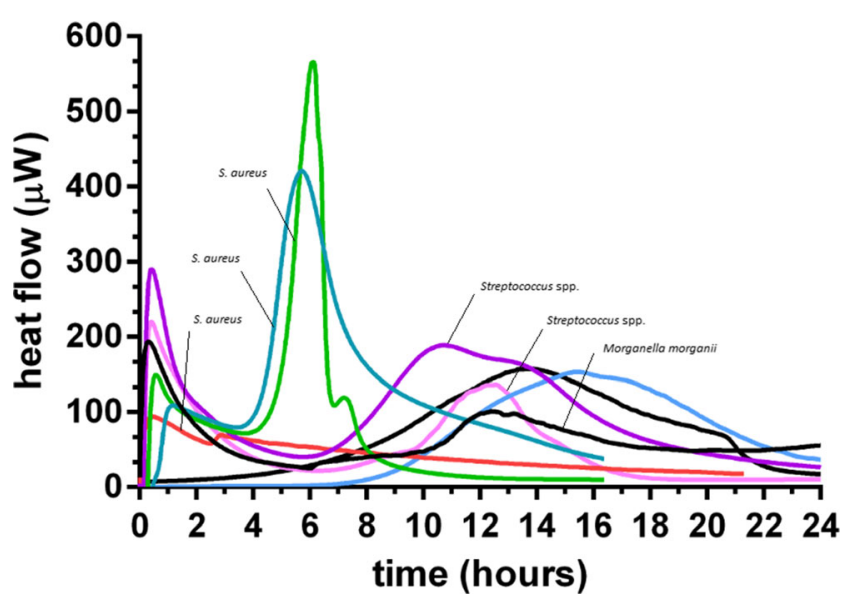

Fig. 1 Heat flow curves of patients with septic arthritis. The microorganisms mentioned are bacteria found on synovial fluid culture instruments) and the price. Once these aspects are improved, the microcalorimeter can soon be a standard method in clinical microbiology [9]. Moreover, the potential of microcalorimetry in antimicrobial susceptibility testing has recently been investigated [11-13]. Braissant and colleagues showed an excellent correlation between microcalorimetry and antimicrobial susceptibility testing using the automated system VITEK ${ }^{\circledR} 2$ system [12]. The results of the studies show promise that microcalorimetry can be used to guide antibiotic therapy in septic arthritis. Another novel diagnostic method that has been evaluated for its improvement in the sensitivity of microbial detection in synovial fluid of septic arthritis is polymerase chain reaction (PCR). The results of these studies were inconclusive. Yang and co-workers [15] used a real-time PCR assay and showed a sensitivity and specificity of 95 and $97 \%$, respectively, compared with bacterial culture of synovial fluid. However, in another study, Bonilla and co-workers showed that only 10 of 16 septic arthritis (sensitivity of $63 \%$ ) were detected correctly [16].

There are several limitations of this study. Firstly, only a small number of patients with septic arthritis were included in this study. Yet, with this small number, this study can already show the significant difference of microcalorimetry results between septic and non-septic arthritis patients. Secondly, the bacteria in the synovial fluid culture were not enumerated and we could not investigate the relation between the bacterial count and the microcalorimetry detection time. However, another study has shown that microcalorimetry can detect bacteria as low as $1 \mathrm{CFU} / \mathrm{ml}[10]$, and we have shown the inverse relation between CFU and the time to positivity [14].

In conclusion, we reported the potential role of microcalorimetry in diagnosing septic arthritis. Microcalorimetry can be used for the accurate and rapid diagnosis of septic arthritis. It has the potential to prompt early initiation of joint lavage and, consequently, lead to improved outcome of septic arthritis, 
and, on the other hand, can prevent unnecessary surgical and antibiotic treatment in non-septic arthritis.

Conflict of interest The authors declare that they do not have any conflict of interest.

Financial disclosure None.

\section{References}

1. Geirsson AJ, Statkevicius S, Víkingsson A (2008) Septic arthritis in Iceland 1990-2002: increasing incidence due to iatrogenic infections. Ann Rheum Dis 67(5):638-643

2. Mathews CJ, Weston VC, Jones A, Field M, Coakley G (2010) Bacterial septic arthritis in adults. Lancet 375(9717):846-855

3. Mathews CJ, Kingsley G, Field M, Jones A, Weston VC, Phillips M, Walker D, Coakley G (2007) Management of septic arthritis: a systematic review. Ann Rheum Dis 66(4):440-445

4. Faraj AA, Omonbude OD, Godwin P (2002) Gram staining in the diagnosis of acute septic arthritis. Acta Orthop Belg 68(4):388-391

5. Goldenberg DL (1998) Septic arthritis. Lancet 351(9097):197-202

6. Söderquist B, Jones I, Fredlund H, Vikerfors T (1998) Bacterial or crystal-associated arthritis? Discriminating ability of serum inflammatory markers. Scand J Infect Dis 30(6):591-596

7. Coutlakis PJ, Roberts WN, Wise CM (2002) Another look at synovial fluid leukocytosis and infection. J Clin Rheumatol 8(2):67-71

8. Margaretten ME, Kohlwes J, Moore D, Bent S (2007) Does this adult patient have septic arthritis? JAMA 297(13):1478-1488
9. Braissant O, Wirz D, Göpfert B, Daniels AU (2010) Use of isothermal microcalorimetry to monitor microbial activities. FEMS Microbiol Lett 303(1):1-8

10. Trampuz A, Salzmann S, Antheaume J, Daniels AU (2007) Microcalorimetry: a novel method for detection of microbial contamination in platelet products. Transfusion 47(9):1643-1650

11. Baldoni D, Hermann H, Frei R, Trampuz A, Steinhuber A (2009) Performance of microcalorimetry for early detection of methicillin resistance in clinical isolates of Staphylococcus aureus. J Clin Microbiol 47(3):774-776

12. Braissant O, Müller G, Egli A, Widmer A, Frei R, Halla A, Wirz D, Gasser TC, Bachmann A, Wagenlehner F, Bonkat G (2014) Seven hours to adequate antimicrobial therapy in urosepsis using isothermal microcalorimetry. J Clin Microbiol 52(2):624-626

13. Furustrand Tafin U, Meis JF, Trampuz A (2012) Isothermal microcalorimetry for antifungal susceptibility testing of Mucorales, Fusarium spp., and Scedosporium spp. Diagn Microbiol Infect Dis 73(4):330-337

14. Borens O, Yusuf E, Steinrücken J, Trampuz A (2013) Accurate and early diagnosis of orthopedic device-related infection by microbial heat production and sonication. J Orthop Res 31(11):1700 1703

15. Yang S, Ramachandran P, Hardick A, Hsieh YH, Quianzon C, Kuroki M, Hardick J, Kecojevic A, Abeygunawardena A, Zenilman J, Melendez J, Doshi V, Gaydos C, Rothman RE (2008) Rapid PCR-based diagnosis of septic arthritis by early Gram-type classification and pathogen identification. J Clin Microbiol 46(4): 1386-1390. doi:10.1128/JCM.02305-07

16. Bonilla H, Kepley R, Pawlak J, Belian B, Raynor A, Saravolatz LD (2011) Rapid diagnosis of septic arthritis using 16S rDNA PCR: a comparison of 3 methods. Diagn Microbiol Infect Dis 69(4):390 395 